\title{
Surgery over lysis in acute pulmonary embolism - let us challenge the guidelines
}

\author{
Marian Zembala ${ }^{1}$, Fryderyk Zawadzki ${ }^{1}$, Marta Wajda-Pokrontka ${ }^{1}, K$ rzysztof Filipiak ${ }^{2}$, Piotr Knapik ${ }^{1}$, \\ Ewa Trejnowska ${ }^{1}$, Michał Zembala ${ }^{1,3}$, Marcin Świerad $^{1}$ \\ ${ }^{1}$ Silesian Centre for Heart Diseases, Zabrze, Poland \\ IIndependent Public Clinical Hospital No. 2, Szczecin, Poland \\ ${ }^{3}$ Pomeranian Medical University, Szczecin, Poland
}

Kardiochir Torakochir Pol 2021; 18 (1): 60-61

We have read with great interest the article prepared by Pruszczyk and Konstantinides published in "Kardiologia Polska" (Kardiol Pol, Polish Heart Journal) [1]. This paper presents strategies for patients with acute pulmonary embolism (PE) depending on the risk of unfavourable prognosis.

Acute PE is still a medical challenge. According to the Silesian Heart and Vessels Database SILCARD from 20062014 , the number of patients with acute PE diagnosis increased almost 2.5-fold in the 8-year follow-up. Hospital mortality in 2006 and 2014 were $17.6 \%$ and $14.4 \%$, respectively, while the 12-month mortality fluctuates around 30\% among patients with acute PE.

Looking at these results, a review of the management of patients with acute pulmonary embolism is needed.

We would like to discuss with the statement contained in the above-cited article about the treatment of haemodynamically unstable patients with acute pulmonary embolism. The authors suggest that systemic thrombolysis should be an in-device method for most high-risk patients, leaving invasive treatments such as transcutaneous catheter therapy and surgical pulmonary embolectomy (SPE) as a second-line therapy, when thrombolysis is contraindicated or failed. That statement is consistent with ESC guidelines for the diagnosis and management of acute pulmonary embolism developed in collaboration with the European Respiratory Society (ERS) from 2019.

It is common knowledge that in acute PE, pressure overload resulting in right ventricular failure is considered a major cause of death. In haemodynamically unstable individuals, rapid and complete revascularization of pulmonary circulation is of the utmost importance.

Based on the experience of the Silesian Centre of Heart Disease, in this group of patients rapid SPE is a better treatment option than systemic thrombolysis.

Recently, a few publications comparing systemic thrombolysis and surgical pulmonary embolectomy have been reported.
Lee et al. [2] performed a retrospective comparison of 2111 adults with acute PE, who underwent either thrombolysis or SPE as a first-line therapy. The authors proved that there were no statistical differences in the 30-day and 5-year mortality in the groups of patients treated with surgical embolectomy or systemic thrombolysis (13.2\% vs. $15.2 \%$; $p=0.62 ; \mathrm{OR}=1.12 ; 95 \% \mathrm{Cl}: 0.72-1.73$ and $13.9 \%$ vs. $27.6 \%$; $\mathrm{HR}=1.11 ; 95 \% \mathrm{Cl}: 0.83-1.49$, respectively). However, systemic thrombolysis significantly increases the risk of reintervention, stroke, and major bleeding. The imperfection of this study is the lack of comparison of the haemodynamic parameters of the analysed patients.

In 2017 Pasrija et al. analysed retrospective medical data from 55 patients who underwent surgical pulmonary embolectomy due to acute PE [3]. The authors showed a significant reduction of right ventricular dysfunction in patients undergoing pulmonary embolectomy, from moderate before to none after surgical embolectomy $(p<0.001)$, as well as high survival rates in the early postoperative time and in a 1-year follow-up (93\% and 91\%, respectively).

Choi et al. [4] raised the subject of outcomes after surgical embolectomy in a review article. Based on the analysis of 32 articles and 936 patients, an in-hospital mortality rate of $16 \%$ with a downward trend in recent years was reported. The most common complication that occured was prolonged ventilation in 33\% of patients (95\% Cl: $21-47)$. The authors also reported changes in systolic pulmonary artery pressure within the preoperative $(57.8 \mathrm{~mm} \mathrm{Hg}, 95 \% \mathrm{Cl}$ : 53-62.7) and postoperative period ( $31.3 \mathrm{~mm} \mathrm{Hg}, 95 \% \mathrm{Cl}$ : 24.9-37.8). In the summary of this review article, the authors suggest that due to improvements of cardiac surgery techniques, surgical embolectomy can be an alternative to systemic thrombolysis in first-line therapy.

Dohle et al. [5] also point out that there is no evidence of a predominance of systemic thrombolysis over surgical embolectomy in high- and intermediate-risk patients. Relying on the data cited by the authors, the mortality rate

Address for correspondence: Prof. Marian Zembala, Department of Cardiac, Vascular, and Endovascular Surgery and Transplantology, Silesian Centre for Heart Diseases in Zabrze, Medical University of Silesia, 9 Curie-Skłodowskiej St, 41-800, Zabrze, Poland, phone: +48 32 3733689,

e-mail: m.zembala@sccs.pl

Received: 22.02.2021, accepted: 6.03.2021. 
is similar between these two methods of treatment, but after surgical embolectomy a smaller amount of diffusion impairment and better right ventricular unloading were observed.

Sharing their view, and taking into consideration the results and possible complications of the therapies as well as the information presented above, we suggest the opinion that the best method of treating haemodynamically unstable patients with acute pulmonary embolism, in whom every minute is invaluable due to the worsening of right ventricular failure, is surgical pulmonary embolectomy.

\section{Conflict of interest}

Michal Zembala - consultant for Abbott Inc., Boston Scientific.

\section{References}

1. Pruszczyk P, Konstantinides S. Where to treat patients with acute pulmonary embolism? Kardiol Pol 2020; 78: 15-19.

2. Lee T, Itagaki S, Chiang YP, Egorova NN, Adams DH, Chikwe J. Survival and recurrence after acute pulmonary embolism treated with pulmonary embolectomy or thrombolysis in New York State, 1999 to 2013. J Thorac Cardiovasc Surg 2018; 155: 1084-1090.

3. Pasrija C, Kronfli A, Rouse M, Raithel M, Bittle GJ, Pousatis S, Ghoreishi M, Gammie JS, Griffith BP, Sanchez PG, Kon ZN. Outcomes after surgical pulmonary embolectomy for acute submassive and massive pulmonary embolism: a single-center experience. J Thorac Cardiovasc Surg 2018; 155: 1095-1106.

4. Choi JH, O'Malley TJ, Maynes EJ, Weber MP, D'Antonio ND, Mellado M, West FM, Galanis T, Gonsalves CF, Marhefka GD, Awsare BK, Merli GJ, Tchantchaleishvili V. Surgical pulmonary embolectomy outcomes for acute pulmonary embolism. Ann Thorac Surg 2020; 110: 1072-1080.

5. Dohle K, Dohle DS, El Beyrouti H, Buschmann K, Emrich AL, Brendel L, Vahl CF. Short- and long-term outcomes for the surgical treatment of acute pulmonary embolism. Innov Surg Sci 2018; 3: 271-276. 